\title{
Results of Few-Nucleon Scattering from Tohoku University and Future Plan
}

\author{
Kimiko Sekiguchi ${ }^{* \dagger}$ \\ Department of Physics, Tohoku University \\ E-mail: kimiko@lambda.phys.tohoku.ac.jp
}

Few-nucleon scattering offers a good opportunities to study dynamical aspects of three-nucleon forces, that are momentum, spin and iso-spin dependent. In this contribution the experimental results of deuteron-proton elastic scattering obtained in the course of the study are presented. The data are compared with the state-of-the art theoretical predictions based on the realistic bare nuclear potentials. Recently the experimental study has been extended to the proton- ${ }^{3} \mathrm{He}$ scattering.

The 9th International workshop on Chiral Dynamics

17-21 September 2018

Durham, NC, USA

* Speaker.

${ }^{\dagger}$ A footnote may follow. 


\section{Introduction}

A hot topic in present day few-nucleon system studies is exploring the properties of threenucleon forces ( $3 \mathrm{NFs})$ that appear when more than two nucleons $(A \geq 3)$ interact. The $3 \mathrm{NFs}$ arise naturally in the standard meson exchange picture in which the main ingredient is considered to be $2 \pi$-exchange between three nucleons along with the $\Delta$-isobar excitation, the mechanism initially proposed a half century ago by Fujita and Miyazawa [1]. Impetus to study 3NFs has also come from chiral effective field theory $(\chi \mathrm{EFT})$ descriptions of nuclear interactions. In that framework consistent two-, three-, and many-nucleon forces are derived on the same footing [2, 3]. The first non-zero contribution to $3 \mathrm{NFs}$ appears in $\chi \mathrm{EFT}$ at the next-to-next-to-leading order $\left(\mathrm{N}^{2} \mathrm{LO}\right)$ of the chiral expansion. That explains why $3 \mathrm{NFs}$ are relatively small compared to $\mathrm{NN}$ forces (2NFs) and why their effects are easily masked. Therefore, it is, in general, hard to find evidence for them.

Few-nucleon scattering offers a good opportunity to study to study dynamical aspects of 3NFs, which are momentum, spin and isospin dependent, since it provides not only cross sections but also a variety of spin observables at different incident nucleon energies. Direct comparison between the experimental data and the rigorous numerical calculations in term of Faddeev theory based on the realistic bare nuclear potentials provides information on 3NFs. The importance of 3NFs in fewnucleon scattering was shown in the nucleon-deuteron $(N d)$ elastic scattering for the first time in Ref. [4]. Clear signals from $3 \mathrm{NFs}$ were found around the cross section minimum occurring at c.m. angle $\theta_{c . m .} \approx 120^{\circ}$ for incident energies above $60 \mathrm{MeV} /$ nucleon. Since then the $p d / n d$ scattering at 60-200 MeV/nucleon have been performed at the facilities, e.g. RIKEN, RCNP, KVI, and IUCF, providing precise data of the cross sections as well as various types of the spin observables [5]. Compilations of recent experiments for $p d$ and $n d$ elastic scattering at intermediate energies are shown in Fig, 1

The four-nucleon $(4 N)$ systems could also play an important role for the study of $3 \mathrm{NFs}$. $3 \mathrm{NF}$ effects are expected to be sizable in the $4 N$ system. In addition, while the $N d$ scattering is essentially a pure isospin $T=1 / 2$ state, tests of the $T=3 / 2$ channel in any $3 \mathrm{NFs}$ can be performed in a $4 N$ system such as proton $-{ }^{3} \mathrm{He}$ scattering. Note importance of study of the iso-spin dependence of $3 \mathrm{NFs}$ has been pronounced for understanding of nuclear system with larger-isospin asymmetry [6, 7]. In recent years, there has been a large progress in solving $4 N$ scattering problem with realistic Hamiltonian even above four-nucleon breakup threshold energies [8, 9], which opens up new possibilities to approaching to properties of $3 \mathrm{NFs}$.

With the aim of exploring the 3 NFs experimental programs of deuteron-proton $(d p)$ scattering as well as proton $-{ }^{3} \mathrm{He}\left(p+{ }^{3} \mathrm{He}\right)$ scattering using the polarized beam and target systems are on going at RIKEN, RCNP Osaka University, and CYRIC Tohoku University, in Japan. In this contribution we introduce recently conducted experiments and present the results of comparison between the experimental data and the theoretical predictions based on the realistic bare nuclear potentials.

\section{Experiments and Results of elastic $d p$ scattering at RIKEN}

The experiments of the $d p$ scattering was performed at the RIKEN Accelerator Facility using the polarized deuteron beams at the incident energies up to $135 \mathrm{MeV} /$ nucleon. Measured observables are the cross section, all deuteron analyzing powers $\left(i T_{11}, T_{20}, T_{21}\right.$, and $\left.T_{22}\right)$, and deuteron to 


\begin{tabular}{|c|c|c|c|c|}
\hline Observable & 10 & 200 & 300 & 400 \\
\hline$\frac{d \sigma}{d \Omega}$ & & ๑०८ • ๑ & & 9 \\
\hline $\begin{array}{ll}\vec{p} & A_{y}^{p} \\
\vec{n} & A_{y}{ }^{n}\end{array}$ & & לְ: & & 9 \\
\hline $\begin{array}{cl}\vec{d} \quad & i T_{11} \\
& T_{20} \\
& T_{22} \\
& T_{21}\end{array}$ & فِ & $\begin{array}{lll}0 & 0 \\
0 & \vdots & \vdots \\
0 & 0 & \vdots \\
0 & 0 & \vdots\end{array}$ & 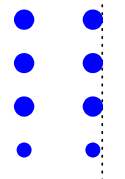 & $\varphi$ \\
\hline $\begin{array}{cc}\vec{p} \rightarrow \vec{p} & K_{y}^{y^{\prime}} \\
& K_{x}^{x^{\prime}} \\
& K_{x}^{z^{\prime}} \\
& K_{z}^{x^{\prime}} \\
& K_{z}^{z^{\prime}}\end{array}$ & & $\pi$ threshold: & $\begin{array}{l}\bullet \\
\bullet \\
\bullet \\
\bullet \\
\bullet\end{array}$ & \\
\hline $\begin{aligned} \vec{d} \rightarrow \vec{p} & K_{y}^{y^{\prime}} \\
& K_{x x}^{y^{\prime}} \\
& K_{y y}^{y^{\prime}} \\
& K_{x z}^{y^{\prime}}\end{aligned}$ & $\bullet$ & $\begin{array}{l}\bullet \\
\bullet \\
\bullet \\
\bullet\end{array}$ & & \\
\hline$\vec{p} \rightarrow \vec{d} \quad K_{y}^{y^{\prime}}$ & & & & $\bullet$ \\
\hline $\begin{array}{ll}\vec{p} \vec{d} & C_{i, j} \\
& C_{i j, \mathbf{k}}\end{array}$ & & 0 & & \\
\hline
\end{tabular}

Figure 1: Compilations of recent experiments of $p d$ and $n d$ elastic scattering at $65-400 \mathrm{MeV} / \mathrm{nucleon}$. Solid circles denote $p d$ experiments and open circles denote $n d$ experiments. The measurements with large circles cover the wide angular range, while those with small circles cover the limited angular range.

proton polarization transfer coefficients $\left(K_{y}^{y^{\prime}}, K_{y y}^{y^{\prime}}, K_{x x}^{y^{\prime}}\right.$, and $\left.K_{x z}^{y^{\prime}}\right)$ [10]. Later the experiments were extended to the RIKEN RI Beam Factory (RIBF). All deuteron analyzing powers were obtained at 190, 250, $294 \mathrm{MeV} /$ nucleon [11, 12,13].

In Fig. 2 some representative experimental results reported in Ref. [13] are shown together with the calculations based on the locally regularized (regulator $R=0.9 \mathrm{fm}$ ) $\mathrm{N}^{4} \mathrm{LO} \chi$ EFT NN potential [14]. Generally the ${ }^{4} \mathrm{LO}$ chiral potential predictions are close to those based on the semiphenomenological NN potentials (e.g. CD Bonn [15], AV18 [16], Nijmegen I and II [17]) [13]. At higher energies, $\mathrm{N}^{4} \mathrm{LO} N \mathrm{~N}$ predictions are generally different from the data. For the cross section large difference is seen at the backward angles, that is quite similar to the results obtained for the semi-phenomenological NN potentials. The vector analyzing power $i T_{11}$ data are well described by the $\mathrm{N}^{4} \mathrm{LO} \chi \mathrm{EFT} \mathrm{NN}$ potential, while large discrepancies are found for the tensor analyzing power $T_{22}$. In order to see how $\chi \mathrm{EFT} 3 \mathrm{NFs}$ describe the data the theoretical treatments are now in progress [18].

\section{Experiments and Results of elastic $p+{ }^{3} \mathrm{He}$ scattering}

Following the experiments of $d p$ scattering we proceed to the experiments of the $p+{ }^{3} \mathrm{He}$ scat- 

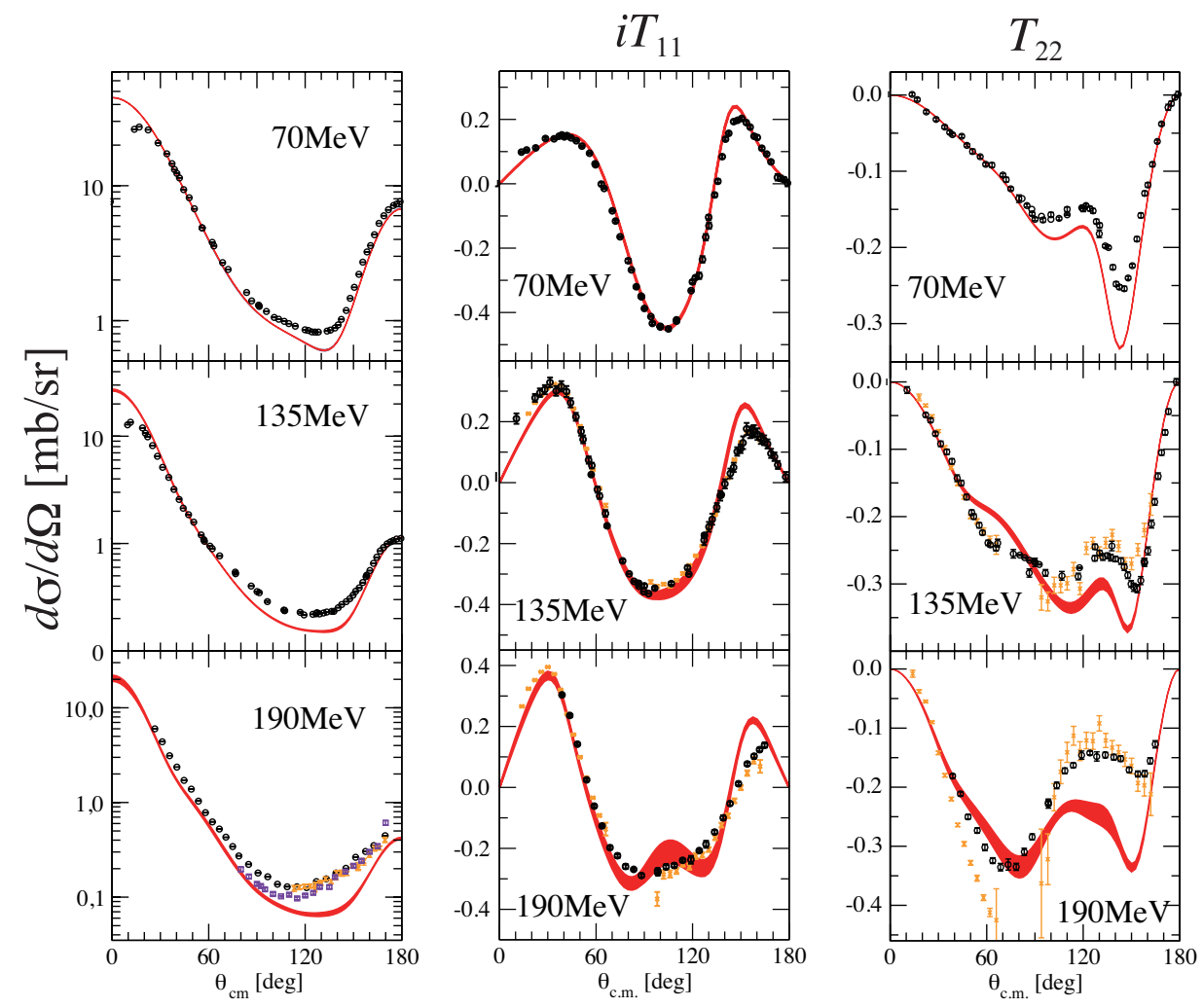

Figure 2: Differential cross section and deuteron analyzing powers $i T_{11}, T_{22}$ for elastic $d p$ scattering. The bands in the figure are the calculations based on the locally regularized (regulator $R=0.9 \mathrm{fm}$ ) $\mathrm{N}^{4} \mathrm{LO} \chi \mathrm{EFT}$ NN potential.

tering at incident nucleon energies above $\sim 65 \mathrm{MeV}$. The experiment consisted of two measurements. The measurement of cross section and proton analyzing power was performed by using $65 \mathrm{MeV}$ polarized proton beam at RCNP, Osaka University. The measurement of ${ }^{3} \mathrm{He}$ analyzing power at an incident proton energy of $70 \mathrm{MeV}$ was performed by using the newly constructed ${ }^{3} \mathrm{He}$ target at Cyclotron Radioisotope Center (CYRIC), Tohoku University. Both measurements covered wide angular range in the center of mass system. In Fig. 3 parts of the data are compared with the rigorous numerical four-nucleon calculations in terms of the Alt, Grassberger, and Sandhas equation based on the modern NN potentials (CDBonn and INOY04 [19]) [20]. The clear discrepancies are found in ${ }^{3} \mathrm{He}$ analyzing power at the angles $80-120$ degrees in the center of mass system. The data analysis is now in progress.

\section{Summary and Outlook}

The few-nucleon scattering provide rich sources to explore the properties of $3 \mathrm{NFs}$ that are momentum, spin and iso-spin dependent. With the aim of exploring the 3NFs experimental programs of deuteron-proton $(d p)$ scattering as well as proton $-{ }^{3} \mathrm{He}\left(p+{ }^{3} \mathrm{He}\right)$ scattering using the polarized beam and target systems are on going at RIKEN, RCNP Osaka University, and CYRIC Tohoku University, in Japan. 

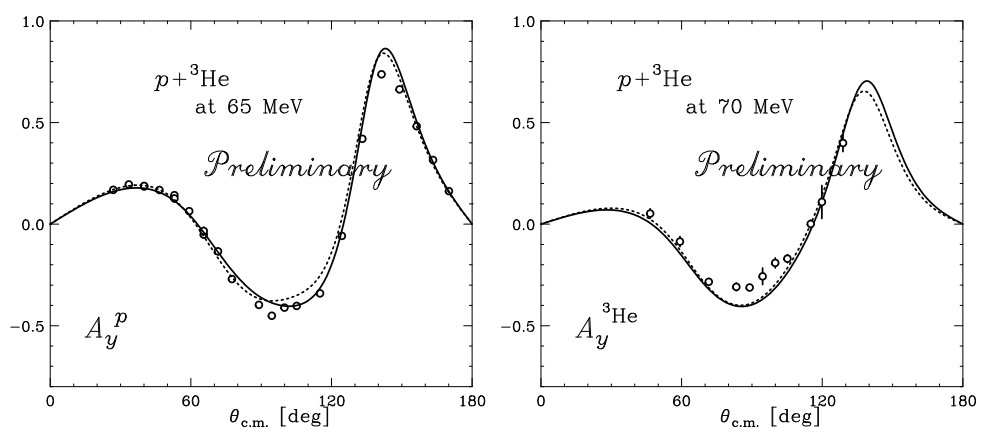

Figure 3: Proton analyzing power and ${ }^{3} \mathrm{He}$ analyzing power for $p+{ }^{3} \mathrm{He}$ elastic scattering. The data of proton analyzing power were taken with a polarized proton beam at $65 \mathrm{MeV}$. Those of ${ }^{3} \mathrm{He}$ analyzing power at 70 $\mathrm{MeV}$ were obtained using a polarized ${ }^{3} \mathrm{He}$ target. The solid (dotted) lines are the calculations based on the INOY04 (CD Bonn) NN potential.

In this contribution, the experimental results obtained with polarized deuteron beams at RIKEN are presented and the recent achievements of theoretical descriptions based on the $\chi \mathrm{EFT}$ NN potential via $d p$ scattering are discussed. The energy and angular dependent results of the cross sections as well as the deuteron analyzing powers show that one needs to take into account chiral 3NFs in future calculations.

The $3 \mathrm{NF}$ effects could also be sizable in the four-nucleon scattering systems in which tests of the isospin $T=3 / 2$ channel in 3 NFs can be performed such as $p+{ }^{3} \mathrm{He}$ scattering. As the first step we conducted the experiments of $p+{ }^{3} \mathrm{He}$ elastic scattering at around $65 \mathrm{MeV}$.

As the next step we are planning the following experiments; i) measurements of $p+{ }^{3} \mathrm{He}$ scattering at $65-200 \mathrm{MeV}$, and ii) measurements of spin correlation coefficients for $d p$ scattering at 65-200 MeV. These data would provide a valuable source to testify nuclear potentials including $3 \mathrm{NFs}$ and/or to determine the low energy constants of the chiral EFT nuclear potentials. Such theoretical work is now in progress [21].

\section{Acknowledgments}

The author would like to thank the collaborators for the experiments performed at RIKEN RI Beam Factory, RCNP Osaka University, and CYRIC Tohoku University. She is also grateful to the strong supports from the theorists, H. Witała, W. Glöckle, H. Kamada, J. Golak, A. Nogga, R. Skibiński, E. Epelbaum, P. U. Sauer, A. Deltuva, and A. C. Fonseca.

\section{References}

[1] J. Fujita and H. Miyazawa, Prog. Theor. Phys. 17, 360 (1957).

[2] U. van Kolck, Phys. Rev. C 49, 2932 (1994).

[3] E. Epelbaum, H.-W. Hammer, U.-G. Meißner, Rev. Mod. Phys. 81, 1773 (2009).

[4] H. Witała et al., Phys. Rev. Lett. 81, 1183 (1998).

[5] See references in N. Kalantar-Nayestanaki et al., Rep. Prog. Phys. 75, 016301 (2012). 
[6] S. C. Pieper et al., V. R. Pandharipande, R. B. Wiringa, and J. Carlson, Phys. Rev. C. 64, 014001 (2001).

[7] S. Gandolfi et al., Eur. Phys. J. A 50, 10 (2014).

[8] A. Deltuva and A. C. Fonseca, Phys. Rev. C 87, 054002 (2013).

[9] M. Viviani, L. Girlanda, A. Kievsky, and L. E. Marcucci, Phys. Rev. Lett. 111, 172302 (2013).

[10] N. Sakamoto et al., Phys. Lett. B 367, 60 (1996); H. Sakai et al., Phys. Rev. Lett. 84, 5288 (2000); K. Sekiguchi et al., Phys. Rev. C 65, 034003 (2002); K. Sekiguchi et al., Phys. Rev. C 70, 014001 (2004); K. Sekiguchi et al., Phys. Rev. Lett 95, 162301 (2005).

[11] K. Sekiguchi et al., Phys. Rev. C 83, 061001(R) (2011).

[12] K. Sekiguchi et al., Phys. Rev. C 89, 064007 (2014).

[13] K. Sekiguchi et al., Phys. Rev. C 96, 064001 (2017).

[14] S. Binder et al., Phys. Rev. C93, 044002 (2016).

[15] R. Machleidt, Phys. Rev. C 63, 024001 (2001).

[16] R. B. Wiringa et al., Phys. Rev. C 51, 38 (1995).

[17] V. G. J. Stoks et al., Phys. Rev. C 49, 2950 (1994).

[18] E. Epelbaum, private communications.

[19] P. Doleschall, Phys. Rev. C 69, 054001 (2004).

[20] A. Deltuva, private communications.

[21] E. Epelbaum et al., Phys. Rev. C 99, 024313 (2019). 